\title{
O Desenvolvimento de Dominância Manual e Podálica em Crianças Destras e Canhotas entre Três a Oito Anos de Idade
}

\author{
William Lee Berdel Martin \\ Universidade Federal do Pará \\ Valéria Pereira Braz Homci \\ Instituto de Estudos Superiores da Amazônia \\ Francisca Morais da Silveira \\ Universidade Federal do Maranhão
}

\begin{abstract}
RESUMO
Essa pesquisa estudou padrões de desenvolvimento de dominância manual, podálica e assimetrias fenotípicas associadas, como dominância mista e a correspondência entre a mão e o pé dominante, em crianças. Pesquisas indicaram que os canhotos diferem dos destros: são mais mistos nas suas preferências manuais e apresentam uma taxa elevada de dominância podálica contralateral, particularmente para chutar bola. Investigou-se em qual faixa de idade a direção e o grau de dominância ficam estáveis; se a dominância podálica se desenvolve simultaneamente com dominância manual e a idade entre canhotos, em que os fenótipos mistos e consistentes emergiram. Crianças $(\mathrm{N}=$ $120)$ de três a oito anos de idade (60 destros e 60 canhotos) foram avaliadas em dez tarefas manuais e quatro podálicas. Em destros e canhotos a direção e o grau de dominância manual estavam estabelecidos aos três anos; em ambos os grupos dominância manual e podálica evoluíram simultaneamente estabelecendo-se na mesma idade, senão antes. Entre canhotos, nos mistos e consistentes, já estava presente aos três anos de idade. A frequência permaneceu uniforme através das demais faixas etárias. Conclui-se que o padrão de dominância neuromotor para tarefas primárias e para o ato de chutar foi invariante, sendo, provavelmente, lateralizado antes de três anos.
\end{abstract}

Palavras-chave: dominância manual; dominância podálica; desenvolvimento lateral; crianças jovens.

\section{ABSTRACT \\ The Development of Handedness and Footedness in Right- and Left-Handed Children Between the age of Three and Eight}

This study focused on the developmental patterns of hand and foot dominance, and associated phenotypic asymmetries, such as mixed dominance and the correspondence between handedness and footedness among young children. Research indicates that for certain lateral traits, left-handers differ from right-handers: they are more mixed in their handedness and express an elevated rate of contralateral foot dominance for ball kicking. We sought to ascertain at which age level the direction and degree of handedness begins to stabilize; and whether footedness develops concurrently with handedness, and among left-handers, at which age do mixed and consistent phenotypes appear. There were 120 children ( 60 dextrals and 60 sinistrals) between the ages of three and eight, all assessed on 10 handedness and four footedness tasks. Results indicated that among both dextrals and sinistrals, the direction and degree of handedness was basically established by the age of three; in both groups handedness and footedness developed simultaneously at the same age, if not before. Among lefthanders, groups of mixed and consistent phenotypes emerged at age three, and their differential frequency remained uniform at ages five and seven. It was concluded that the pattern of neuromotor dominance for primary manual tasks and for ball kicking was relatively invariante, probably lateralized prior to three-years of age.

Keywords: handedness; footedness; lateral development; young children.

Tarefas unimanuais que requerem força muscular (e.g., arremessar uma bola, serrar, martelar com preci- são e jogar tênis) e/ou coordenação fina e movimentos bem sincronizados da mão e dos dedos (e.g., escrever, 
desenhar, recortar com uma faca) tendem a ser mediadas por áreas específicas no sistema neuromotor do hemisfério contralateral à mão que está executando a atividade (Geschwind \& Galaburda, 1987; Hecaen, 1984; Wilson, 1998). Em comparação, tarefas simples que podem ser desempenhadas facilmente com qualquer mão, tais como apanhar objetos, descartar baralhos e pentear cabelo, entre outras, tendem a ser associadas com atividade bilateral envolvendo ambos os hemisférios. A primeira classe foi denotada de "tarefas primárias" e a segunda de "tarefas secundárias" ou de "preferências" (cf. Annett, 1985, 2002).

Os resultados de experimentos recentes sobre lateralidade usando técnicas de neuroimagem, de modo geral, reforçaram uma distinção enfatizada desde o trabalho de Broca no século 19, que diz existirem diferenças nítidas entre destros e canhotos no padrão de lateralização cerebral para funções motoras (Amunts e cols., 2000; Beaton, 2004; Jäncke \& Steinmetz, 2004; Peters, 2000). Por exemplo, quando destros escrevem com a mão direita, a excitação neuronial ocorre predominantemente (embora não exclusivamente) na rede motora do hemisfério esquerdo (Garry e cols., 2004; Rijntjes e cols., 1999; Volckman e cols., 1998). Ao contrário, quando canhotos escrevem com a mão esquerda o padrão de ativação ocorre mais no hemisfério direito, embora o mesmo tenda a ser mais variável e difuso, estendendo-se até o hemisfério oposto (Kawashima e cols., 1997; Klöpel e cols., 2007; Singh e cols., 1998; Volckman e cols., 1998).

Ao nível fenotípico, diferenças entre canhotos e destros na direção e no grau de dominância e na proficiência manual têm sido amplamente registradas, mais especificamente:

(a) Em resposta às tarefas contidas em inventários de preferências manuais, em relação aos destros os canhotos quase sempre exibem um alto nível de variabilidade, que por sua vez decorre do maior número de canhotos que apresentam um perfil misto nas suas preferências (e.g. um indivíduo que escreve, martela e serra com a mão esquerda, e arremessa, joga ping pong e recorta com a mão direita); o que por sua vez reflete dissociação lateral (e.g. uma atividade controlada principalmente pelo hemisfério esquerdo e outra pelo hemisfério direito), mais especificamente, entre 25 e $30 \%$ dos canhotos preferem arremessar uma bola - com força e precisão com a mão direita, enquanto apenas 1 a $2 \%$ dos destros mostrou mais proficiência com a mão esquerda (Gilbert \& Wysocki, 1992; McManus e cols., 1999).

(b) Quando a dominância manual e podálica foram avaliadas em conjunto (usando o pé para chutar como a referência), a direção das duas especializações mostrou uma correspondência unilateral. Isto é, por volta de $90 \%$ da população foi ipsilateral (e.g. destros que chutam um pênalti com o pé direito), com a prevalência de preferência contralateral e ambilateral presente nos demais 10\% (Peters, 1988; Porac \& Coren, 1981). Novamente, como grupo, os canhotos desviam radicalmente desta tendência geral; em contraste com $5 \%$ dos destros, quase $33 \%$ dos canhotos chutam bola (no caso de um pênalti) com o pé contralateral. Além do mais, por volta de $60 \%$ dos canhotos mistos, em particular aqueles que arremessam com a mão direita, também preferem chutar com o pé direito, enquanto que a grande maioria dos canhotos consistentes prefere chuta bola com o pé esquerdo (Machado, 2002; Martin \& Machado, 2005; Martin \& Porac, 2007; Peters, 1991, 1996).

Quando se trata da maturação destas assimetrias laterais, a sequência ontogenética de dominância manual tem sido amplamente investigada. Segundo os dados de estudos mais antigos, a dominância manual estava completamente estabelecida até a idade de 10 ou 11 anos (Gesell \& Ames, 1947; Hildreth, 1948, 1949). Estes autores, no entanto, não diferenciaram entre a direção (direito ou esquerdo) e o grau de dominância, tipicamente indexado por um escore manual. Atualmente existe certo consenso de que a direção começa a ficar mais evidente e definida entre dois e meio e quatro anos de idade; por outro lado ainda há discordância quanto ao período em que o grau começa a ficar estável e consistente (Dellatolis e cols., 1997; Dellatolis \& De Agostini, 2004; Manoel \& Connolly, 1998; Provins, 1992; Xentien e cols., 1991).

Apesar da quantidade de dados gerados no decorrer dos últimos 60 anos em pesquisas com bebês e crianças jovens, com referência à natureza ontogenética do processo de lateralização cerebral, subjacente às dominâncias laterais, nenhuma teoria tem recebido ampla aceitação pela comunidade científica. Existem, atualmente, duas teorias alternativas e controvertidas. A primeira, a hipótese de "equipotência", ou de lateralização progressiva, postulava que até a idade de dois anos a linguagem ou a dominância manual pode ser mediada por ambos os hemisférios. Posteriormente, uma função se tornaria mais assimétrica gradati- 
vamente e alcançaria maturidade somente na fase da puberdade (Corballis \& Morgan, 1978; Lennenberg, 1967). No entanto, a evidência obtida sobre os efeitos de lesões nos dois hemisférios e da plasticidade cerebral em crianças apontou para uma interpretação contraditória, a de que a assimetria funcional já está completa desde o nascimento. Esta segunda teoria, a de "invariância", enfatiza que os precursores de dominância manual já estariam programados dentro do sistema nervoso central, mesmo sem ser claramente evidenciado no comportamento até a idade de dois a três anos (Hiscock, 1988; Hiscock \& Kinsbourne, 1995; Iaccino, 1993). Atualmente, no campo da neuropsicologia do desenvolvimento, versões da teoria de invariância tendem a predominar sobre o esquema postulando equipotência (Hahn, 1987; Iaccino, 1993).

Considerando-se somente os dados de pesquisas com crianças canhotas e destras na faixa etária a partir dos três anos de idade, os resultados foram contraditórios. Alguns apontaram para um padrão de variabilidade até a idade de seis anos, tanto em destros quanto em canhotos (De Agostini e cols., 1992; Fennell e cols., 1983; Longini \& Orsini, 1988). Outros achados indicaram que o grau de dominância dos canhotos estabilizava antes dos destros (McManus e cols., 1988; Özturk e cols., 1999). Em outras investigações houve tendências inversas: os destros exibiram um grau consistente de destrimanismo no intervalo entre três a cinco anos, enquanto os canhotos não demonstraram nenhum padrão uniforme, mesmo antes da idade de nove anos (Auzias, 1990; Bruml, 1972; Bryden e cols., 2007; Gudmundsson, 1993).

Com referência à sequência evolutiva da correspondência entre preferência podálica $(\mathrm{PP})$ e manual (PM), a informação disponível é ambígua. Não está claro se o padrão de PP estabiliza-se antes ou depois da PM ou se as duas ficam estabelecidas ao mesmo tempo. Também não está claro se os canhotos diferem dos destros, em particular quando se leva em conta o grau reduzido de consistência e a frequência de contralateralidade característica de canhotos. De acordo com Belmont e Birch (1963), a direção da PP para chutar em destros e canhotos estava basicamente estabelecida a partir da idade de cinco anos, enquanto a PM demorou mais dois anos para tornar-se estável. Gabbard e seus colaboradores (1991) contestaram esta conclusão. Com base nos resultados de uma série de pesquisas com indivíduos na faixa entre três a 20 anos, Gentry e Gabbard (1995) inferiram que, em relação a PM, a dominância podálica ficou variável somente até a faixa entre 11 e 12 anos. Por outro lado o procedimento classificatório usado por Gabbard e colaboradores foi questionável porque o mesmo confundiu a direção com o grau das duas assimetrias. Nas análises eles alocaram destros e canhotos inconsistentes em uma amostra "amorfa" de mistos, sem referência à dominância genérica da mão e na contagem não separaram os dados para as três tarefas podálicas usadas - chutar bola, pisar com força e tracejar letras -, embora "chutar" tenda a ser atividade mais familiar e fidedigna do que "pisar" e "tracejar" (Martin e cols., 2004; Peters, 1996).

Em um trabalho anterior, Homci (2005) identificou estas limitações e procurou verificar se de fato as diferenças fenotípicas encontradas entre canhotos e destros variaram significativamente em crianças com três, cinco e sete anos de idade. Em termos de direção os dados apontaram para um perfil de invariância. Especificamente entre canhotos a percentagem de contralateralidade manual e podálica, bem como preferência mista (houve poucos destros nestas categorias), foi equivalente em cada faixa etária. A única diferença significativa emergiu quando o grau de preferência manual e podálica foram avaliados. Através das faixas etárias o escore médio da mão e do pé era consistentemente para o lado direito em destros, enquanto o desempenho dos canhotos era muito variável e uma preferência média para o pé esquerdo somente apareceu em crianças com idade de sete anos.

Homci (2005) reconheceu que a validade e generalidade destes resultados foram limitadas porque o tamanho da amostra de cada faixa etária foi relativamente pequeno. $\mathrm{Na}$ presente pesquisa foi possível dobrar o número de participantes em cada grupo, aumentando assim o poder estatístico. Por extensão, este estudo visa averiguar (1) se a direção e o grau de canhotismo desenvolveram-se antes do destrimanismo; (2) se a dominância mista desenvolveu-se depois ou concomitantemente com dominância consistente; e (3) se, em termos da direção e do grau, preferência podálica, ipsilateral e contralateral, desenvolveu-se antes ou depois da preferência manual. No fim, avaliamos à medida que as tendências encontradas em destros e canhotos aderiram às duas teorias alternativas.

\section{MÉTODO}

\section{Amostras}

A amostra foi constituída de 120 crianças, 60 destras e 60 canhotas, distribuídas em três faixas etárias distintas: três, cinco e sete anos. Houve 20 destros e 20 canhotos (com 10 meninos e 10 meninas) em cada grupo. 
Todos foram alunos recrutados de escolas do ensino pré-escolar e fundamental da rede particular e pública nas cidades de Belém, Pará e São Luis, Maranhão.

\section{Medidas e Materiais}

O Teste de Preferências Laterais em Crianças (TPLC): Dominância Manual

O TPLC foi desenvolvido por nós, com base no modelo de De Agostini \& Dellatolas (2001), com algumas alterações nos itens. $\mathrm{O}$ teste foi divido em três partes. A primeira era composta de um questionário com dados pessoais da criança, inclusive a dominância manual geral. Foram as professoras que identificaram as crianças destras e canhotas e posteriormente a classificação foi verificada em entrevista com a mãe ou responsável além de nossas próprias observações. Na segunda parte tínhamos o teste de preferência manual composto de 10 tarefas unimanuais: escrever num quadro branco pequeno, apagar o quadro branco, apertar parafusos, martelar pregos, recortar com tesoura um desenho, arremessar uma bola, comer com colher, jogar tênis de praia, pentear o cabelo e escovar os dentes. Com a exceção do quadro branco e a tesoura, todos os objetos foram brinquedos plásticos escolhidos de acordo com a recomendação de De Agostini e cols. (1992) para serem facilmente manipulados por crianças pequenas e para evitar respostas bimanuais. Cada criança foi avaliada individualmente e houve três tentativas em dias diferentes. Assim, as respostas foram quantificadas segundo os seguintes valores: $+1=$ uso preferencial da mão direita e $-1=$ uso preferencial da mão esquerda. Tal procedimento, com três tentativas para cada uma das 10 tarefas, gerou um escore manual entre +30 e -30 pontos. Desta maneira as crianças eram consideradas dominantes da mão direita para uma dada tarefa se pelo menos duas das três tentativas se realizaram com a mão direita (i.e. um escore de $2-1=+1$ ); caso contrário a dominância era da mão esquerda. Este procedimento era rigoroso porque o mesmo eliminou a possibilidade de uma criança ser classificada como "ambimanual" numa dada tarefa. Na prática, em tarefas primárias, tais como "martelar", "arremessar uma bola" e "chutar um pênalti", notamos que algumas crianças "experimentaram" com a mão não dominante em uma ou mais tentativas, embora fosse fácil identificar qual mão ou pé era mais proficiente. Nestes casos, houve explicações adicionais e as tentativas foram repetidas. A fim de diferenciar a preferência consistente da preferência mista, adotamos o critério usado por Peters (1991). Consideramos destros consis- tentes aqueles que preferiram a mão direita para realizar todas as 10 tarefas e destros mistos aqueles que preferiram a mão esquerda para uma ou mais das 10 tarefas. Os canhotos consistentes deveriam preferir a mão esquerda para nove ou dez tarefas, enquanto canhotos mistos deveriam preferir a mão direita para ao menos duas tarefas. O critério mais liberal para um canhoto ser classificado como consistente foi baseado na suposição de Peters (1991) de que a convivência em ambientes físicos organizados para a maioria destra deve levar um número substancial de canhotos consistentes a executar pelo menos uma atividade rotineira com a mão direita.

\section{O Teste de Preferências Laterais em Crianças: Dominância Podálica}

A terceira parte do TPLC contava com quatro tarefas podálicas: chutar um pênalti, pisar com força, tracejar números no chão e apagar os números. Semelhantemente à preferência manual, as crianças foram testadas individualmente em uma sala organizada para esta atividade e solicitamos a realização da tarefa com o pé preferido. Houve três tentativas em dias diferentes para cada tarefa e usamos os mesmos valores (de +1 a -1) para indicar a direção da preferência. Neste caso o escore podálico total variou entre +12 e -12 pontos. Na classificação da correspondência mão e pé (ipsilateral ou contralateral), o pé preferido para chutar um pênalti serviu como a tarefa de referência. Se a criança mostrou uma preferência para o pé direito em pelo menos duas das três tentativas, sua dominância podálica foi considerada no lado direito. Se, em pelo menos duas das três tarefas, a criança preferiu executar a tarefa com o pé esquerdo, então a dominância podálica foi classificada como sendo no lado esquerdo. Com testagem direta, no caso de "chutar um pênalti", foi possível identificar, com pouca dúvida, o pé mais proficiente (i.e. aquele que, mesmo não sendo bem coordenado, chutava a bola dentro do alvo no chão com força e precisão). Em contraste, as outras três tarefas não eram bem conhecidas ou praticadas pelas crianças e o uso de um pé em duas tentativas e o outro pé em uma tentativa ocorreu frequentemente.

\section{Procedimento}

As medidas citadas acima foram aplicadas em crianças previamente identificadas pelos professores, coordenadores e ultimamente pelas mães e responsáveis como destros e canhotos. A participação de todas as crianças foi autorizada pela mãe ou responsável e a primeira parte foi preenchida junto com a mesma. As 
avaliações nos testes manuais e podálicas foram realizadas em salas disponíveis em cada escola. As crianças foram testadas também em duas medidas de habilidade motora: o Tabuleiro de Annett e o Teste de Pontilhar Pontinhos, embora os resultados destas medidas sejam apresentados num trabalho separado (Martin, Homci \& Silveira, 2009).

\section{RESULTADOS}

\section{Tendências Fenotípicas Gerais}

Os dados pertinentes às tendências gerais na presente amostra constam na Tabela 1. Não identificamos nenhuma diferença entre os sexos nas distribuições e por este motivo analisamos as frequências combinadas. Poucos destros mostraram dominância cruzada $(5 \%)$ e preferência mista $(8,3 \%)$. Em contraste com os destros, ficou evidente que os canhotos foram bem mais mistos na sua dominância manual $(30 \%$ vs. $8,3 \%): \chi^{2}(1, N=120)=9,09, p=0,005$. Em termos de correspondência entre a mão e o pé, os canhotos foram seis vezes mais contralaterais no pé preferido para chutar um pênalti que os destros $(31,7 \%$ vs. $5 \%)$ : $\chi^{2}(1, N=120)=14,25, \mathrm{p}<0,001$. Dentro do grupo de canhotos $50 \%$ dos mistos preferiram chutar com o pé contralateral versus $23,8 \%$ dos canhotos consistentes: $\chi^{2}(1, \mathrm{~N}=60)=3,99, \mathrm{p}=0,046$. O único outro trabalho que apresentou os dados totais para a correspondência mão/pé entre crianças nesta mesma faixa etária foi o de De Agostini \& Dellatolas (2001). Os índices de contralateralidade relatados foram significativamente maiores do que os nossos: no caso dos destros $(12,8 \%$ vs. $5,0 \%)$ e dos canhotos $(53 \%$ vs. $31,7 \%)$. Levando em conta que, como De Agostini e Dellatolas, usamos testagem direta e o mesmo critério de apuração, as divergências possivelmente decorreram de diferenças no tamanho das amostras $(\mathrm{N}=755$ crianças franceses vs $\mathrm{N}=120$ nesta investigação), do delineamento de pesquisa (i.e. longitudinal vs. transversal) e da falta de controle pelos pesquisadores franceses sobre respostas aleatórias ou "experimentais". Por outro lado as tendências no presente estudo foram semelhantes àquelas obtidas em investigações com crianças de sete anos (Clymer \& Silva, 1985; Nachson e cols., 1983) e acima (Martin \& Machado, 2005; Martin, Machado \& Paixão, 2004; Martin \& Porac, 2007).

\section{TABELA 1}

Frequência de Correspondência Ipsilateral e Contralateral para Chutar e Consistência Manual em Destros e Canhotos Segundo o Gênero

\begin{tabular}{|c|c|c|c|c|c|}
\hline \multirow[b]{2}{*}{ Grupo } & \multirow[b]{2}{*}{$\mathrm{N}$} & \multicolumn{2}{|c|}{ Correspondência } & \multicolumn{2}{|l|}{ Consistência } \\
\hline & & Ipsilateral & Contralateral & Consistente & Misto \\
\hline Destros & 30 & $28(93,3)$ & $02(06,7)$ & $26(86,7)$ & $04(13,3)$ \\
\hline Destras & 30 & $29(96,7)$ & $01(03,3)$ & $29(96,7)$ & $01(03,3)$ \\
\hline Total & 60 & $57(95,0)$ & $03(05,0)$ & $55(91,7)$ & $05(08,3)$ \\
\hline Canhotos & 30 & $21(70,0)$ & $09(30,0)$ & $18(60,0)$ & $12(40,0)$ \\
\hline Canhotas & 30 & $20(66,7)$ & $10(33,3)$ & $24(80,0)$ & $06(20,0)$ \\
\hline Total & 60 & $41(68,3)$ & $19(31,7)$ & $42(70,0)$ & $18(30,0)$ \\
\hline
\end{tabular}

Nota. Percentagens entre parênteses.

A Tabela 2 apresenta a direção das respostas dos destros e canhotos em cada tarefa manual e podálica. Embora tabeladas na forma de frequência, os escores de cada item das 120 crianças foram submetidos a uma análise de fidedignidade psicométrica. O coeficiente alfa $(\alpha)$ de Cronbach indicou níveis altos de consistência interna: nas 10 atividades manuais, $\alpha=$ 0,98 e nas quatro podálicas, $\alpha=0,95$. Apesar do nível satisfatório de precisão psicométrica, apontamos para algumas tendências quanto à direção de respostas em cada grupo. Como de praxe, os destros apresentaram o percentual maior de assimetria manual e podálica, com apenas 1 a $3 \%$ preferindo a mão esquerda em cinco das dez tarefas. Segundo nossas observações, a tarefa "apertar um parafuso" gerou mais incerteza, posto que $15 \%$ das crianças tentaram a usar ambas as mãos ao mesmo tempo. 
TABELA 2

Frequências e Percentagens da Dominância dos Membros Direitos (MD) e Esquerdos (ME) para Cada Tarefa Manual e Podálica

\begin{tabular}{lllll}
\hline & \multicolumn{2}{l}{ Destros $(\mathrm{N}=60)$} & \multicolumn{2}{l}{ Canhotos $(\mathrm{N}=60)$} \\
\cline { 2 - 4 } Tarefas & $\mathrm{MD}$ & $\mathrm{ME}$ & $\mathrm{MD}$ & $\mathrm{ME}$ \\
\hline Escrever no Quadro & $60(100)$ & $0(00,0)$ & $00(00,0)$ & $59(98,3)$ \\
Apagar 0 Quadro & $59(98,3)$ & $1(01,7)$ & $21(35,0)$ & $60(100)$ \\
Apertar Parafuso & $57(95,0)$ & $5(05,0)$ & $06(10,0)$ & $39(65,0)$ \\
Martelar Prego & $59(98,3)$ & $1(01,7)$ & $13(21,7)$ & $54(90,0)$ \\
Usar Tesoura & $59(98,3)$ & $1(01,7)$ & $16(26,7)$ & $47(78,3)$ \\
Arremessar Bola & $60(100)$ & $0(00,0)$ & $02(03,3)$ & $44(73,3)$ \\
Comer com Colher & $59(98,3)$ & $1(01,7)$ & $12(20,0)$ & $58(96,7)$ \\
Jogar Tênis & $60(100)$ & $0(00,0)$ & $09(15,0)$ & $48(80,0)$ \\
Pentear Cabelo & $59(98,3)$ & $1(01,7)$ & $06(10,0)$ & $51(85,0)$ \\
Escovar Dentes & $58(96,7)$ & $2(03,3)$ & $19(31,7)$ & $54(90,0)$ \\
Chutar Pênalti & $57(95,0)$ & $3(05,0)$ & $16(26,7)$ & $41(68,3)$ \\
Pisar com Força & $56(93,3)$ & $4(06,7)$ & $16(26,7)$ & $44(73,3)$ \\
Tracejar Números & $59(98,3)$ & $1(01,7)$ & $12(20,0)$ & $44(73,3)$ \\
Apagar Números & $58(96,7)$ & $2(03,3)$ & & $48(80,0)$ \\
\hline
\end{tabular}

Nota. Percentagens entre parênteses.

Em comparação, foram os canhotos que manifestaram a maior taxa de preferência com a mão oposta. Neste grupo, $35 \%$ apertaram o parafuso com a mão direita e semelhante aos destros, cerca de $15 \%$ manipularam o objeto com ambas as mãos. Foi interessante notar que quase $27 \%$ preferiram arremessar uma bola com a mão direita, mas dos poucos estudos que avaliaram a mão para arremessar em crianças pré-escolares e acima (Brito e cols, 1992; Gudmundsson, 1993), nenhum apresentou os dados separadamente para destros e canhotos. Não obstante a taxa obtida concordou basicamente com os dados presentes em pesquisas com canhotos mais velhos, onde, com uma exceção (Brito \& Santos-Morales, 1999), as respostas foram obtidas por meio de questionários em vez de testagem direta (cf. Gilbert \& Wysocki, 1992; McManus e cols., 1999; Peters, 1996). Além do mais, descobrimos uma diferença significativa relacionada ao gênero. Enquanto $40 \%$ dos canhotos arremessaram com a mão direita, apenas $13 \%$ das canhotas manifestaram esta preferência: $\chi^{2}(1, \mathrm{~N}=60)=5,46, \mathrm{p}=0,02$. Nenhuma pesquisa citada acima relatou a presença de tal padrão de divergência entre os sexos para esta tarefa e, sem dados de outros estudos realizados com crianças na mesma faixa etária, a generalidade deste achado é duvidosa.
O Padrão de Dominância Lateral e Assimetrias Fenotípicas Através das Idades

Avaliamos em primeiro lugar a sequência de desenvolvimento dos traços fenotípicos principais. Entre destros a consistência manual e correspondência ipsilateral entre a mão e o pé começaram a ser fortemente lateralizada para o lado direito desde três anos de idade. Poucos destros preferiram lidar com a mão e o pé esquerdo e a assimetria direita permaneceu invariante nas três faixas etárias para todas as tarefas manuais e podálicas.

Embora os canhotos tenham sido mais variáveis na sua dominância, o percentual classificado como "mistos" foi uniforme, ao nível de $30 \%$ em todas as três idades e no caso de chutar um pênalti a mesma consistência foi verificada. Nas 10 tarefas manuais, uma avaliação daquelas consideradas como primárias (Martelar, escrever, arremessar, jogar tênis), também revelaram um padrão estável. No caso de arremessar uma bola, por exemplo, entre as crianças com três anos de idade, $25 \%$ arremessaram com a mão direita e naquelas com sete anos a prevalência era $30 \%$. Uma análise das demais tarefas secundárias, de modo geral, mostrou pouca mudança através das faixas etárias, com a única exceção sendo "apertar um parafuso". Foi encontrada uma tendência, embora não significativa, para a preferência esquerda aumentar à medida que a idade aumentou de $60 \%$ (três anos de idade) para $75 \%$ (sete anos de idade). 
No mesmo grupo, nas tarefas podálicas, enquanto "chutar um pênalti" se mostrou estável desde três de idade, nos demais três itens a direção da preferência foi menos uniforme. Enquanto 30 a $35 \%$ das crianças canhotas entre três e seis anos preferiram o pé direito, a taxa caiu para 10 a $15 \%$ na faixa de sete anos. Novamente atribuímos este padrão de instabilidade à natureza ambígua destas últimas três tarefas. De modo geral, ficou evidente que muitas das crianças mais jovens tiveram pouca experiência com cada atividade e houve muita experimentação onde, em média, $40 \%$ preferiram um pé em uma tentativa e o outro pé nas demais duas tentativas.

Neste ponto avaliamos a presença de possíveis mudanças no grau médio das preferências ao longo dos três níveis de idade. Assim as médias e os desvios padrão para cada tarefa, em cada grupo, foram resumidos na Tabela 3.

TABELA 3

Médias do Escore Manual e Podálico Através das Idades e do Gênero

\begin{tabular}{|c|c|c|c|c|c|}
\hline \multirow[b]{2}{*}{ Idade/Gênero } & \multirow[b]{2}{*}{$\mathrm{N}$} & \multicolumn{2}{|c|}{ Escore Manual } & \multicolumn{2}{|c|}{ Escore Podálico } \\
\hline & & $\mathrm{M}$ & $\mathrm{DP}$ & $\mathrm{M}$ & $\mathrm{DP}$ \\
\hline \multicolumn{6}{|c|}{ Destros $(N=60)$} \\
\hline \multicolumn{6}{|l|}{ Três Anos } \\
\hline Masculino & 10 & 26,70 & 2,95 & 08,60 & 4,01 \\
\hline Feminino & 10 & 29,30 & 1,34 & 11,00 & 1,94 \\
\hline Total & 20 & 28,00 & 2,60 & 09,80 & 3,30 \\
\hline \multicolumn{6}{|l|}{ Cinco Anos } \\
\hline Masculino & 10 & 26,80 & 7,05 & 10,40 & 2,27 \\
\hline Feminino & 10 & 28,90 & 2,03 & 10,40 & 4,40 \\
\hline Total & 20 & 27,85 & 5,16 & 10,40 & 3,41 \\
\hline \multicolumn{6}{|l|}{ Sete Anos } \\
\hline Masculino & 10 & 24,40 & 8,04 & 09,40 & 3,13 \\
\hline Feminino & 10 & 28,80 & 2,15 & 11,40 & 1,35 \\
\hline Total & 20 & 26,60 & 6,16 & 10,40 & 2,56 \\
\hline \multicolumn{6}{|c|}{$\underline{\text { Canhotos }}(\mathrm{N}=60)$} \\
\hline \multicolumn{6}{|l|}{ Três Anos } \\
\hline Masculino & 10 & $-18,80$ & 08,30 & $-2,10$ & 8,77 \\
\hline Feminino & 10 & $-20,30$ & 14,59 & $-6,20$ & 9,77 \\
\hline Total & 20 & $-19,55$ & 11,58 & $-4,15$ & 9,28 \\
\hline \multicolumn{6}{|l|}{ Cinco Anos } \\
\hline Masculino & 10 & $-19,10$ & 9,72 & $-7,00$ & 08,48 \\
\hline Feminino & 10 & $-20,80$ & 12,80 & $-1,40$ & 10,50 \\
\hline Total & 20 & $-19,95$ & 11,10 & $-4,20$ & 08,97 \\
\hline \multicolumn{6}{|l|}{ Sete Anos } \\
\hline Masculino & 10 & $-21,00$ & 08,96 & $-8,00$ & 4,22 \\
\hline Feminino & 10 & $-24,20$ & 09,77 & $-6,40$ & 7,35 \\
\hline Total & 20 & $-22,60$ & 09,27 & $-7,20$ & 5,88 \\
\hline
\end{tabular}

As análises foram efetuadas separadamente para os destros e canhotos porque a maioria dos escores estava distribuída em lados opostos do ponto zero teórico e quaisquer comparações entre os dois grupos sempre resultaram em diferenças altamente significativas. Com referência aos dados para destros os escores manuais foram submetidos à ANOVA fatorial 2 (sexo) x 3 (idade). O único efeito significativo emer- giu para sexo: $\mathrm{F}(1,54)=6,20, \mathrm{p}<0,02$. Através das três idades (e nos totais), as destras, em média, manifestaram um grau mais forte de destrimanismo do que os destros $(M=28,8$ vs. $M=24,4)$. Além do mais, segundo o teste de Levine $\left(\mathrm{F}_{\text {Levine }}\right)$ para igualdade entre as variâncias (ver os valores dos DPs na Tabela 3) em relação às destras, os destros exibiram um alto grau de variabilidade, principalmente nas crianças 
com cinco e sete anos: $F_{\text {Levine }}(1,58)=9,50, p<0,004$. Os escores podálicos também foram avaliados através da ANOVA 2 (sexo) x 3 (idade) e nenhum efeito principal ou interação foi significativo. Novamente os destros demonstraram maior variabilidade do que as destras, principalmente nas idades de três e sete anos: $\mathrm{F}_{\text {Levine }}(1,58)=5,25, \mathrm{p}<0,03$.

Entre os canhotos os dados também foram sujeitos a ANOVA 2 (sexo) x 3 (idade). No caso do escore manual não foi encontrado nenhum efeito significativo para sexo ou idade. Embora o escore médio das canhotas tendesse a ser maior do que os canhotos em todas as faixas etárias, nenhuma divergência foi significativa. A análise subsequente do escore podálico também não acusou nenhum resultado significativo, diferente do escore manual houve tendências descontínuas entre os sexos, de uma idade para outra, mas novamente nenhuma diferença fidedigna emergiu. De modo geral os escores dos canhotos foram muito dispersos em torno da média (ver os DPs na Tabela 3 ). Em comparação com os destros o desempenho dos canhotos foi significativamente heterogêneo: no caso de preferência manual, $F_{\text {Levine }}(1,118)=32,43$, $\mathrm{p}<0,001$, e para preferência podálica, $\mathrm{F}_{\text {Levine }}(1,118)$ $=55,58, \mathrm{p}<0,001$. Estes resultados, no entanto, já eram previstos, posto que o alto grau de variação para estes traços é característico de canhotos.

Por final, considerando os dados combinados, a correlação entre o escore manual e podálica foi altamente significativa: $\mathrm{r}(118)=0,814, \mathrm{p}<0,001$. Este nível de associação concordou aproximadamente com o valor relatado do único estudo que apresentou correlações para os dois traços entre crianças préescolares e acima (Coren, Porac \& Duncan, 1981). Por outro lado não encontramos nenhuma correlação significativa entre idade, os escores manuais e podálicos, através dos dois grupos e nos totais. Este resultado sugere que apesar da presença de variabilidade (principalmente entre os meninos destros e os canhotos) entre a idade de três e oito anos, não houve nenhum aumento substancial no grau de assimetria manual e podálico.

\section{DISCUSSÃO}

$\mathrm{Na}$ introdução diferenciamos a direção do grau de dominância manual, bem como tarefas primárias e secundárias, com a finalidade de esclarecer as variações tipicamente observadas em crianças jovens, destras e canhotas, e evitar problemas terminológicos. Por exemplo, em alguns estudos uma criança que sempre desenha com a mão esquerda, arremessa e empilha objetos com a mão direita tem sido caracterizada como tendo "dominância indefinida", ou "fraca" (Auzias, 1990; Bruml, 1972; Hildreth, 1948), ou até "ambimanual" (Gentry \& Gabbard, 1995; Gudmundsson, 1993), quando o padrão de desempenho seria melhor descrito como "misto", no sentido de favorecer uma mão para algumas tarefa e a outra mão para outras (Peters, 1991, 1996). Semelhante a Homci (2005), questionamos a validade do referente "ambidestro", pois em crianças na faixa etária entre três e sete anos às vezes não está claro se tal padrão de dupla preferência realmente reflete proficiência igualada entre as mãos ou uma tendência para experimentar com cada mão quando há duas ou mais tentativas nas mesmas atividades durante a avaliação. Para obter uma visão mais clara preferimos eliminar a possibilidade de respostas ambilaterais e examinar as assimetrias laterais em cada tarefa ao longo das idades. Além do mais, ao contrário das outras investigações com crianças préescolares, dominância consistente e mista foi definida de antemão com base no número de atividades executadas com cada mão.

Com referência à direção, entre os destros, a dominância para a mão direita nas tarefas primárias e mesmo na maioria das secundárias foi relativamente consistente ao longo das três idades. Em contraste, houve muito mais canhotos apresentando dominância mista, cerca de $30 \%$, e esta tendência foi uniforme em todas as faixas etárias; por este motivo o traço não deve ser considerado como um indicador de "imaturidade" motora ou cognitiva como alguns autores alegaram (Smirni \& Zappalá, 1989; Tan, 1985). Quando a direção lateral de cada uma das 10 tarefas foi analisa$\mathrm{da}$, independentemente da natureza, a frequência das preferências mudou pouco em função da idade. A tendência geral concordou com os achados de Gudmondsson (1993), mas não com aqueles de Brito e cols. (1992). Estes últimos pesquisadores relataram uma diminuição na frequência de preferência mista entre a idade de quatro a sete anos. É possível que esta discordância decorra de diferenças na instrumentação. Em vez de testagem direta eles eliciaram as preferências por meio de um questionário.

No que se refere à direção de dominância podálica, levando em conta a falta de familiaridade com as outras atividades, usamos "chutar pênalti" como referência. Entre os destros a ipsilateralidade foi constante entre três e oito anos de idade e entre os canhotos, apesar da maior taxa de contralateralidade, não houve nenhuma oscilação significativa através das três ida- 
des. Este resultado sugere que esta forma de dissociação lateral estava presente nos canhotos entre a idade de dois a três anos; ou seja, o traço talvez reflita uma disposição biológica, não sendo influenciado unicamente por fatores sociais. Em suma não houve nenhuma indicação de que a dominância podálica nessa tarefa ficou estabelecida depois da dominância manual, como Gentry \& Gabbard (1995) argumentaram; ao contrário, parece que as duas especializações emergiram simultaneamente em destros e canhotos, mesmo antes da idade de três anos.

Quanto ao grau de dominância manual entre destros, as médias do escore manual foi mais alta entre as destras do que os destros em todas as faixas etárias. Existem dados de outras pesquisas em que, em comparação com os destros, as destras demonstraram, constantemente, um desempenho mais assimétrico para o lado direito; no entanto, esses dados foram obtidos somente em algumas pesquisas com adultos, enquanto a maioria das pesquisas não acusou nenhuma diferença entre os gêneros (Annett, 2002; Dellatolas \& De Agostini, 2004). Por outro lado não encontramos este tipo de diferença em nenhuma investigação com crianças jovens. É possível, no entanto, que este resultado foi fortuito devido a erros aleatórios. Em comparação, em canhotos a presença de um número de crianças com dominância mista resultou num nível maior de variação e médias reduzidas. Apesar do menor nível de assimetria, a tendência média foi uniforme nas três faixas etárias e, semelhante aos destros, nos totais as correlações não foram significativas, indicando a ausência de qualquer relação unilinear. Neste caso, as conclusões de McManus e cols. (1988) e de Özturk e cols. (1999), indicando que o grau de dominância desenvolveu-se mais rápido em canhotos, não foram sustentadas.

Por fim, o esquema teórico de equipotencialidade, apresentado na introdução e que postula a dominância cerebral para funções motoras que progridem de um estado não diferenciado (até dois a três anos de idade) até um estado assimétrico e definitivo na fase de adolescência, não é apoiado pelos dados obtidos nessa pesquisa. Nossos achados indicaram que até a idade de três anos a dominância manual era relativamente estável em destros e canhotos, com a possibilidade de mudanças futuras na direção e no grau de preferências secundárias decorrentes da influência de eventos sociais e ambientais. Em resumo, o padrão de resultados concordou mais com a teoria de invariância relativa e a suposição de que assimetrias laterais desdobram-se de precursores programados desde a primeira infância.

\section{REFERÊNCIAS}

Amunts, K., Jäncke, L., Mohlberg, H., Steinmetz, H., \& Stilles, K. (2000). Interhemispheric asymmetry of the human motor cortex related to handedness and gender. Neuropsychologia, 38, 304-312.

Annett, M. (1985). Left, right, hand and brain: The right shift theory. London: Lawrence Erlbaum.

Annett, M. (2002). Handedness and brain asymmetry: The right shift theory. East Sussex: Psychology Press.

Auzias, M. (1990). Niños diestros, niños zurdos. Madrid: Visor.

Beaton, A. A. (2004). The nature and determinants of handedness. Em K. Hugdahl \& R. J. Davidson (Orgs.), The asymmetrical brain (pp. 105-158). Cambridge, MA: MIT Press.

Belmont, L. \& Birch, H. G. (1963). Lateral dominance and rightleft awareness in normal children. Child DevelopmentI, 34, 257-270.

Brito, G. N. O., Lins, M. F. C., K. Paumgartten, F. J. R., \& Brito, L. S. O. (1992). Hand preference in 4- to 7-year-old children: An analysis with the Edinburgh Inventory in Brazil. Developmental Neuropsychology, 8, 59-68.

Brito, G. N. O. \& Santos-Morales, T. R. (1999). Lateral preferences in 8- to15-year old Brazilian children assessed with the Edinburgh Inventory: Different measures of handedness and comparison with younger children and adults. Developmental Neuropsychology, 16, 433-453.

Bruml, H. (1972). Age changes in preference and skill measures of handedness. Perceptual and Motor Skills, 34, 3-14.

Bryden, P. J., Roy, E. A., \& Spence, J. (2007). An observational method of assessing handedness in children and adults. Developmental Neuropsychology, 32, 825-846.

Clymer, P. E. \& Silva, P. A. (1985). Laterality, cognitive ability and motor performance in a sample of seven-year olds. Journal of Human Movement Studies, 11, 59-68.

Corballis, M. \& Morgan, J. M. (1978). On the biological basis of human laterality I. Evidence for a maturational left-right gradient. Behavioral and Brain Sciences, 2, 261-336.

Coren, S., Porac, C., \& Duncan, P. (1981). Lateral preference behaviors in preschool children and young adults. Child Development, 52, 443-450.

De Agostini, M. \& Dellatolas, G. (2001). Lateralities in normal children ages 3 to 8 and their role in cognitive performances. Developmental Neuropsychology, 20, 429-444.

De Agostini, M., Pare, C., Goudot, D., \& Dellatolas, G. (1992). Manual preference and skill development in preschool children. Developmental Neuropsychology, 8, 41-57.

Dellatolas, G., \& De Agostini, M. (2004). Développement et plasticité manualle chez l'enfant. Em J. Fagard (Org.), Droitiers/Gauchers: Dês asymétries dans tous lês sens (pp. 7179). Marseille: Solal Editeurs.

Dellatolas, G., Teubert-Bitter, P., Curt, F., \& De Agostini, M. (1997). Evolution of degree and direction of hand preference in children: Methodological and theoretical issues. Neuropsychological Rehabilitation, 7, 387-399.

Fennell, E. B., Satz, P., \& Morris, R. (1983). The development of handedness and dichotic ear listning asymmetries in relation to school achievement: A longitudinal study. Brain and Language, $35,248-262$. 
Gabbard, C., Dean, M., \& Haensley, P. (1991). Foot behavior during early childhood. Journal of Applied Developmental Psychology, 12, 131-137.

Garry, M. I., Kamen, G., \& Nordstrom, M. A. (2004). Hemispheric differences in the relationship between corticomotor excitability changes following a fine motor task and motor learning. Journal of Neurophysiology, 91, 1570-1578.

Gentry, V., \& Gabbard, C. (1995). Foot-preference behavior: A developmental perspective. Journal of General Psychology, $122,37-45$.

Gesell, A., \& Ames, L. B. (1947). The development of handedness. Journal of Genetic Psychology, 70, 155-175.

Geschwind, N., \& Galaburda, A. (1987). Cerebral lateralization: Biological mechanisms, associations, and pathology. Cambridge, MA: Bradford.

Gilbert, A., \& Wysocki, C. J. (1992). Hand preference and age in the United States. Neuropsychologia, 30, 601-608.

Gudmundsson, E. (1993). Lateral preference of preschool and primary school children. Perceptual and Motor Skills, 77, 819828.

Hahn, W. K. (1987). Cerebral lateralization of function: From infancy through childhood. Psychological Bulletin, 101, 376392.

Hécaen, H. (1984). Lês gauchers. Paris: Presses Universitaires de France.

Hildreth, G. (1948). Manual dominance in nursery school children. Journal of Genetic Psychology, 72, 29-45.

Hildreth, G. (1949). The development and training of hand dominance: II. Developmental tendenceis in handedness. Journal of Genetic Psychology, 75, 221-254.

Hiscock, M. (1988). Behavioral asymmetries in normal children. Em D. L. Molfese \& S. J. Segalowitz (Orgs.), Brain lateralization in children (pp. 85-160). New York: Guilford.

Hiscock, M., \& Kinsbourne, M. (1995). Phylogeny and ontogeny of cerebral lateralization. Em R. J. Davidson \& K. Hugdahl (Orgs.), Brain asymmetry (pp. 535-578). Cambridge, MA: MIT Press.

Homci, V. P. B. (2005). A ontogênese de dominância manual, podálica e habilidades manuais em crianças destras e canhotas entre três a oito anos de idade. Dissertação de mestrado não-publicada, Universidade Federal do Pará. Belém.

Iaccino, J. F. (1993). Left brain-right brain differences: Inquiries, evidence and new approaches. Hillsdale, NJ: Lawrence Erlbaum.

Jäncke, L. \& Steinmetz, H. (2004). Anatomical brain asymmetries and their relevance for functional asymmetries. Em K. Hugdahl \& R. J. Davidson (Orgs.), The asymmetrical brain (pp. 187229). Cambridge, MA: MIT Press.

Kawashima, R., Inoue, K., Satgo, K., \& Fukuda, H. (1997). Functional asymmetry of cortical motor control in left-handed subjects. NeuroReport, 8, 1729-1732.

Klöppel, S., Vongerichten, A., Eimeren, T. V., Frackowiak, R. S. J., \& Siebner, H. R. (2007). Can left-handedness be switched? Insights from an early switch of handwriting. Journal of Neuroscience, 27, 7847-7853.

Lennenberg, E. (1967). The biological foundations of language. New York: John Wiley.
Longini, A. M., \& Orsini, L. (1988). Lateral preferences in preschool children: A research note. Journal of Child Psychiatry and Psychology, 29, 533-539.

Machado, A. H. (2002). Desempenho em tarefas do tempo compartilhado em destros e canhotos com preferência podálica ipsilateral e contralateral. Dissertação de mestrado não-publicada, Universidade Federal do Pará, Belém.

Manoel, E. J., \& Connolly, K. J. (1998). The development of manual dexterity in young children. Em K .J. Connolly (Org.), The psychobiology of the hand (pp. 177-198). London: MacKeith Press.

Martin, W. L. B., Homici, V., \& Silveira, F. M. (2009). Circledotting performance across the age span among right- and lefthanded Brazilians. Manuscrito em preparação.

Martin, W. L. B., \& Machado, A. H. (2005). Deriving estimates of contralateral footedness from prevalence rates in samples of Brazilian right- and left-handers. Laterality, 10, 353-368.

Martin, W. L. B., Machado, A. H., \& Paixão, C. B. (2004). Footedness across the age span among Brazilian right- and lefthanders. Perceptual and Motor Skills, 99, 999-1006.

Martin, W. L. B., \& Porac, C. (2007). Patterns of handedness and footedness in switched and non-switched Brazilian lefthanders: Cultural effects on the development of lateral preferences. Developmental Neuropsychology, 31, 159-179.

McManus, I. C., Porac, C., Bryden, M. P., \& Boucher, R. (1999). Eye dominance, writing hand and throwing hand. Laterality, 4, 173-192.

McManus, I. C., Silk, G., Cole, D. R., Mellon, A. F., Wong, J., \& Kloss, J. (1988). The development of handedness in children. British Journal of Developmental Psychology, 6, 257-273.

Nachson, I., Denno, D., \& Aurand, S. (1983). Lateral preference of hand, eye and foot: Relation to cerebral dominance. International Journal of Neurosciences, 18, 1-10.

Özturk, C., Durmazier, N., Ural, B., Karaagaoglu, E., Yalatz, K., \& Anlar, B. (1999). Hand and eye preference in normal preschool children. Clinical Pediatrics, 38, 677-680.

Peters, M. (1988). Footedness: Asymmetries in foot preference, skill, and neuropsychological assessment of foot movement. Psychological Bulletin, 103, 179-192.

Peters, M. (1991). Laterality and motor control. Em G. R. Bock \& J. Marsh (Orgs.), Biological asymmetry and handedness (pp. 300-308). New York: John Wiley.

Peters, M. (1996). Hand preference and performance in lefthanders. Em D. Elliott \& E. A. Roy (Orgs.), Manual asymmetry in motor performance (pp. 99-120). Boca Raton, FL: CRC.

Peters, M. (2000). Contributions of imaging techniques to our understanding of handedness. Em M. K. Mandal, M. B. Bulman-Fleming \& F. Tiwari (Orgs.), Side bias: A neuropsychological perspective (pp. 191-224). Dordrecht: Kluwer.

Porac, C., \& Coren, S. (1981). Lateral preferences and human behavior. Heidelberg: Verlag.

Provins, K. A. (1992). Early infant motor asymmetries and handedness: A critical evaluation of the evidence. Developmental Neuropsychology, 8, 325-365.

Rijntjes, M., Dettmers, C., Buchel, C., Kiebel, S., Frackowiak, R. S. J., \& Weiller, C. (1999). A blueprint for movement: 
Functional and anatomical representations in the human motor system. Journal of Neuroscience, 19, 8043-8048.

Singh, I., Takahahi, S., Kurihara, N., Furuta, S., \& Tamura, H. (1998). Comparison of ispilateral activation between right and left-handers: A functional MR study. NeuroReport, 9, 18611866.

Smirni, P., \& Zappalá, G. (1989). Manual behavior, lateralization of manual skills and cognitive performance of preschool children. Perceptual and Motor Skills, 68, 267-272.

Tan, L. E. (1985). Laterality and motor skills in four-year-olds. Child Development, 56, 119-124.

Volckmann, J., Schnitzler, A., Witte, O. W., \& Freund, H. (1998). Handedness and asymmetry of hand representation in human motor córtex. Journal of Neurophysiology, 79, 2149-2154.
Wilson, F. R. (1998). The hand: How its use shapes the brain, language, and human culture. New York: Pantheon.

Xentian, L., Minggao, L., Huikun, G., \& Kuihe, J. (1991). Experimental study of grasping with right or left hand by children six months to three years old. International Journal of Mental Health, 20, 12-26.

\section{Sobre os autores:}

Recebido: $23 / 04 / 2008$ Última revisão: 03/04/2009 Aceite final: 04/05/2009

William Lee Berdel Martin: PhD. Professor do Núcleo de Teoria e Pesquisa do Comportamento da UFPA.

Valéria Pereira Braz Homci: Mestre em Teoria e Pesquisa do Comportamento pela UFPA. Professora do Instituto de Estudos Superiores da Amazônia.

Francisca Morais da Silveira: Doutora em Teoria e Pesquisa do Comportamento pela UFPA. Professora do Departamento de Psicologia da Universidade Federal do Maranhão.

Endereço para correspondência: wlbmartin@gmail.com. 